\title{
Germanica
}

\section{De quelques lectures de Schnitzler dans la critique consacrée à Eyes Wide Shut}

Reading Schnitzler through french critic Reviews of Eyes Wide Shut

Schnitzlers Dartellungen in den Rezensionen von Eyes Wide Shut

\section{Audrey Giboux}

\section{(2) OpenEdition}

\section{Journals}

Édition électronique

URL : http://journals.openedition.org/germanica/2130

DOI : $10.4000 /$ germanica. 2130

ISSN : 2107-0784

Éditeur

Université de Lille

Édition imprimée

Date de publication : 15 juin 2013

Pagination : 133-145

ISBN : 9782913857315

ISSN : 0984-2632

\section{Référence électronique}

Audrey Giboux, "De quelques lectures de Schnitzler dans la critique consacrée à Eyes Wide Shut »,

Germanica [En ligne], 52 | 2013, mis en ligne le 01 octobre 2013, consulté le 06 octobre 2020. URL http://journals.openedition.org/germanica/2130 ; DOI : https://doi.org/10.4000/germanica.2130

(c) Tous droits réservés 


\title{
De quelques lectures de Schnitzler dans la critique consacrée à Eyes Wide Shut
}

\author{
Audrey GIBOUX \\ Université Blaise Pascal, Clermont-Ferrand \\ CRLC de l'université Paris-Sorbonne
}

\section{Schnitzler et la France : un siècle de malentendus}

La diffusion de l'œuvre de Schnitzler en France s'est révélée erratique tout au long du $\mathrm{XX}^{\mathrm{e}}$ siècle ; comme l'a montré Karl Zieger, son image dans la critique s'est fixée autour de topö̈ qui ne donnent qu'une vision superficielle, voire erronée de son œuvre : il est considéré comme le représentant d'une Jeune Vienne caricaturée dans sa légèreté souriante et sentimentale, rythmée de valses, comme un « maître de la petite forme », un « Maupassant autrichien », ou un épigone littéraire de Freud - lectures qui négligent la variété, l'universalité et la modernité de son œuvre ${ }^{1}$.

Plusieurs éléments ont partiellement rééquilibré cette image réductrice : une vague de (re)traductions, après la mort, en 1981, de Suzanne Clauser alias Dominique Auclères, titulaire des droits exclusifs de la traduction de son œuvre ; le succès, en 1986, de l'exposition du Centre Pompidou Vienne 1880-1938 : l'apocalypse joyeuse, qui suscite un enthousiasme nouveau pour cet auteur ; l'inscription de La Nouvelle

1. - Karl Zieger, Enquête sur une réception. Arthur Schnitzler et la France 18941938, Villeneuve d'Ascq, Presses universitaires du Septentrion, 2012. 
rêvée (Traumnovelle) au programme de littérature comparée "Fictions de l'intime » de l'agrégation de Lettres en 2001-2003, etc. Le film Eyes Wide Shut de Kubrick, adaptation controversée de la Traumnovelle, constitue quant à lui un épisode paradoxal de la réception schnitzlérienne en France : en rendant le nom de l'inspirateur de Kubrick plus familier au public français ${ }^{2}$, la réception du film semble provoquer de nouveaux malentendus dans la représentation de Schnitzler par la critique journalistique et cinématographique.

\section{Schnitzler jeté aux oubliettes?}

Lors de la sortie française du film, le 15 septembre 1999, peu après le décès du cinéaste survenu le 7 mars, les rumeurs les plus sulfureuses circulent sur cette œuvre jalousement gardée secrète. La critique est alors très partagée. Dans nombre des premières recensions, les allusions à Schnitzler sont ponctuelles, quand elles ne font pas totalement défaut. Pour ne citer qu'un exemple révélateur, Gilles Verdiani assassine l'œuvre sans même en mentionner l'inspiration schnitzlérienne ${ }^{3}$. Michel Boujut parle d'un film «dans le formol », d'une «viennoiserie qui n'est porteuse d'aucun vertige, d'aucun trouble », incapable de sonder la « pénombre des âmes » dont Schnitzler faisait l'analyse ${ }^{4}$. Dans une critique admirative ( $\mathrm{La}$ tentation du risque »), Claude Baignères se contente de mentionner que le scénario « s'inspire d'un roman de Schnitzler $»^{5}$. Dans un article intitulé « 1999, l'odyssée du sexe et de l'angoisse », Serge Kaganski loue quant à lui la beauté plastique du film, avant de citer lapidairement la nouvelle dans la version américaine du traducteur Otto P. Schinnerer, utilisée par Kubrick : «Eyes Wide Shut est très fidèlement adapté de Rhapsody, une nouvelle [...] d'Arthur Schnitzler, dans laquelle l'auteur autrichien évoquait les aventures sexuelles fantasmées par les deux membres d'un couple ${ }^{6}$. Tout aussi factuel est le court résumé que Lætitia Mailhes donne de ce film «basé sur le roman de l'auteur autrichien Arthur Schnitzler, Traumnovelle », décrit comme « une odyssée où rêve et réalité se mêlent en un fascinant jeu d'échecs » (« Tom Cruise et Nicole Kidman droit dans les yeux »)7.

2. - Le Monde mentionne la réédition de la traduction de Dominique Auclères accompagnée du scénario du film chez Pocket, n¹6996, 17 septembre 1999, p. VIII ; voir de même Positif, n464, octobre 1999, p. 137.

3. - Première, $\mathrm{n}^{\circ} 270$, septembre 1999, p. 69. Philippe Fraisse, qui dans Le Cinéma au bord du monde. Une approche de Stanley Kubrick fait d'Eyes Wide Shut une odyssée de l'homme moderne aliéné, évacue de même l'enjeu schnitzlérien, Paris, Gallimard, «L'Infini », 2010.

4. - Charlie Hebdo, $\mathrm{n}^{\circ} 378,15$ septembre 1999, p. 15.

5. - Le Figaro, $\mathrm{n}^{\circ} 17135,15$ septembre 1999, p. 28.

6. - Les Inrockuptibles, $\mathrm{n}^{\circ} 209$, du 25 au 31 août 1999, p. 12-14.

7. - L'Humanité, nº17083, 19 juillet 1999, p. 24. 
La qualité et la pertinence de l'adaptation du texte à l'écran sont parfois mises en question. François Forestier, dans son dossier « Kubrick fin de party », affiche sa déception quant à ce «thriller sexuel » qu'il juge désérotisé, soulignant que « du roman d'Arthur Schnitzler, Traumnovelle, il ne reste pas grand-chose $»^{8}$. La recension élogieuse due à Samuel Blumenfeld, « Le mystère du couple ou l'enfer selon Kubrick », salue pourtant succinctement l'efficacité de l'adaptation : « Tourné à Londres et situé dans un New York reconstitué à la perfection, [le film crée] un onirisme qui sied bien à Rien qu'un rêve, la nouvelle de Schnitzler adaptée par Kubrick $»^{9}$. Pascale Ferran, réalisatrice de la version française d'un film qu'elle dépeint comme une « expérience de laboratoire » sur l'influence de l'imaginaire sur le réel, témoigne de son extrême fidélité à l'esprit de l'intrigue schnitzlérienne ${ }^{10}$.

Mais cet argument, aussi invoqué à charge contre le réalisateur ${ }^{11}$, est retourné par Philippe Garnier dans son article « Venise ouvre l'œil. La Mostra démarre avec Eyes Wide Shut », qui incrimine le " jésuitisme endémique » de cette œuvre de « cinéma-éprouvette » :

Quel homme, en 1999, se verrait catapulté dans pareil abîme de doute et de jalousie, simplement parce que sa femme lui avoue avoir fantasmé sur un autre ? Même Schnitzler, en 1926, avait besoin de transposer son histoire [quelques années plus tôt], la pensant déjà dépassée. [...] Schnitzler était un voyeur et un sensualiste ; Kubrick ni l'un ni l'autre ${ }^{12}$.

La critique d'Olivier Séguret, «L'œil castré », prolonge ironiquement l'analyse du caractère prétendument daté de la jalousie du héros apprenant les fantasmes de sa femme :

Le profond retournement déceptif que produit le film vient [...] de son sujet. De la matière inventée par Schnitzler dans son [sic] Traumnovelle, Kubrick rapporte un scénario en deux phases. Premièrement, le jeune William Harford [...] comprend un jour que sa femme Alice est susceptible d'avoir des fantasmes et c'est pour lui la fin du monde. Deuxièmement, dès l'instant où il laisse à son tour ouverte

8. - Le Nouvel Observateur, ${ }^{\circ} 1818$, du 9 au 15 septembre 1999, p. 12-14.

9. - Le Monde, $\mathrm{n}^{\circ} 16994,15$ septembre 1999, p. 32.

10. - «Les yeux grand fermés : à propos du doublage de Eyes Wide Shut », entretien avec Colette Milon et Jean-Philippe Renouard, disponible sur : Vacarme, $\mathrm{n}^{\circ} 10$, hiver 2000, http://www.vacarme.org/article874.html. Voir aussi la recension de Jean-Claude Loiseau, Télérama, n²592, du 18 au 24 septembre 1999, p. 32 et celle de Jean-Paul Enthoven, Le Point, n' ${ }^{\circ}$ 1408, 10 septembre 1999, p. 103.

11. - Voir la critique de François Gorin, Télérama, n²584, du 24 au 30 juillet 1999, p. 29.

12. - Libération, $\mathrm{n}^{\circ} 5688,1^{\text {er }}$ septembre 1999, p. 29. 
la porte de son imaginaire sexuel, il va être happé dans un engrenage hallucinant $[\ldots]^{13}$.

Comment expliquer cette réduction de la Traumnovelle à un prétexte éculé ?

\section{Schnitzler et Kubrick, objets de controverses}

Ce sujet semblait pourtant crucial aux yeux du cinéaste, qui avait acheté les droits d'adaptation de la nouvelle dès le début des années 1970. Son hommage à Schnitzler est vibrant dans cet entretien accordé à Michel Ciment en 1972 :

il y a le roman d'Arthur Schnitzler que j'ai toujours l'intention d'adapter. [...] Le livre oppose les aventures réelles d'un mari et celles oniriques de sa femme, et il pose la question : y a-t-il une différence sérieuse entre rêver une aventure sexuelle et en avoir une ? [...] Je pense que c'est l'une des plus belles histoires de Schnitzler et l'une de celles où il y a le plus d'imagination. Je suis un grand admirateur de son œuvre ${ }^{14}$.

La lecture dépréciative de la nouvelle est en fait véhiculée par l'équipe du film, qui assure sa promotion après la mort du cinéaste, bien que son épouse et son producteur exécutif insistent sur la force du texte schnitzlérien et sur l'interprétation optimiste qu'en donnait Kubrick, qui avait envisagé d'en tirer une comédie ${ }^{15}$. Leon Vitali, son assistant, avoue : "Je n'aurais jamais pensé qu'il allait faire de cette nouvelle de Schnitzler quelque chose d'aussi imposant ${ }^{16}$. Le coscénariste du film, Frederic Raphael, est encore bien davantage responsable du dénigrement de l'œuvre de Schnitzler. En 1999, il fait paraître un récit de sa collaboration avec Kubrick ; intitulé Eyes Wide Open, ce témoignage à la tonalité polémique est traduit en français la même année ${ }^{17}$. Raphael y explique que Kubrick lui avait donné pour seul précepte : « Je veux faire l'histoire d'Arthur, mais à New York, de nos jours ». Mais le scénariste n'a que mépris pour la Traumnovelle; il évoque la «faiblesse »d'une histoire « démodée et très européenne », au finale « un peu trop gentil », sans « véritable progression ».

13. - Libération, $\mathrm{n}^{\circ} 5700,15$ septembre 1999, p. 33.

14. - Michel Ciment, Kubrick, édition définitive, préface de Martin Scorsese, Paris, Calmann-Lévy, 2004, p. 156.

15. - « Brève rencontre. Christiane Kubrick et Jan Harlan », entretien accordé à Yann Tobin et Laurent Vachaud, Positif, Spécial Kubrick, n464, octobre 1999, p. 41-44.

16. - Les Inrockuptibles, $\mathrm{n}^{\circ} 209$, du 25 au 31 août 1999, p. 22.

17. - Frederic Raphael, Deux ans avec Kubrick, par le scénariste de Eyes Wide Shut, traduction de Richard Cunningham, Paris, Plon, 1999. 
Face au désir de Kubrick de «transposer l'action de ce Vienne finde-siècle au New York d'aujourd'hui », pour hisser le propos du film à un niveau archétypal et universel, Raphael, plus intéressé par la question de la judéité commune à Schnitzler et Kubrick, se montre dubitatif : « des tas de choses n'ont-elles pas changé depuis 1900, à commencer par les relations hommes-femmes ? Vous croyez ? me dit Kubrick. Moi, je ne crois pas ». Le procès en désuétude du sujet trouve pourtant son origine dans ces pages, et se cristallise selon Raphael, peu informé des relations véritables entre Freud et Schnitzler, dans l'enjeu des rêves érotiques racontés par les personnages, passages qu'il juge " niais », " prétentieux » et « surécrits »; et de dépeindre le nouvelliste en médiocre avatar du fondateur de la psychanalyse :

ces rêves $-[\ldots]$ c'était nouveau quand Freud l'était aussi. Ça ne me paraît pas très convaincant. Je me demande ce que Sigmund en aurait pensé. Pas grand-chose, j'imagine. Tous ces dialogues, cette précision dans les souvenirs, c'est tellement... littéraire... L'auteur doit avoir lu Freud, non?

Mais Kubrick se montre inflexible face à ces critiques, et n'a de cesse d'enjoindre Raphael de « suivre le rythme d'Arthur ${ }^{18}$.

Le témoignage de Raphael, abondamment relayé par la presse française, semble néanmoins occulter l'admiration de Kubrick pour Schnitzler ${ }^{19}$. Si l'œuvre schnitzlérienne est ainsi parfois conçue comme un simple prétexte, incapable de donner lieu à un film intéressant, ou, selon les partis-pris esthétiques des critiques, sublimé par un film considéré comme un chef-d'œuvre testamentaire, il se dégage aussi une tendance contraire, qui voit dans le film, alors jugé vulgaire, une trahison de l'auteur autrichien. L'idée de la supériorité du livre sur le film est sensible dans une critique très négative de Philippe Garnier, «Le dernier Kubrick à première vue »; le film, « édulcoré », qu'il estime très inférieur au cinéma d'Ophuls, lui semble un contresens infligé au sujet schnitzlérien, qui perd, en raison d'un anachronisme ici invoqué au discrédit de Kubrick, sa force subversive :

si Kubrick colle d'étonnamment près au texte d'Arthur Schnitzler, il le lit mal ou choisit de s'en écarter quand ça l'arrange [...]. Il y avait pourtant des choses chez Schnitzler sacrément plus intéressantes [...]. Et ce qui au temps de Schnitzler était réellement scandaleux (on ne parlait

18. - Ibid., p. 35, 38, 47, 62, 69, 79, 97, 130.

19. - Voir notamment Première, $\mathrm{n}^{\circ} 269$, août 1999, p. 66-73 ; Libération, Un été 1999, n5684, 27 août 1999, p. VI-VII ; Le Nouvel Observateur, n¹818, du 9 au 15 septembre 1999, p. 20-22 ; L'Express, n²514, du 9 au 15 septembre 1999, p. 84 ; Télérama, $\mathrm{n}^{\circ} 2592$, du 18 au 24 septembre 1999, p. 36-38; Les Cahiers du cinéma, n539, octobre 1999 , p. 7-8, etc. 
pas de ces choses-là entre époux) devient un peu incompréhensible, voire risible, dans le monde d'aujourd'hui ${ }^{20}$.

L'éloge de Schnitzler contre Kubrick se radicalise chez certains spécialistes de l'auteur autrichien : si la présentation de Pierre Deshusses de sa version de la Traumnovelle trouve dans la notoriété du film un argument pour relire la nouvelle ${ }^{21}$, la préface de Brigitte Vergne-Cain et Gérard Rudent à la traduction de Philippe Forget apparaît comme un réquisitoire contre un Kubrick accusé de massacrer un chef-d'œuvre classique ; cette critique prolonge le débat récurrent sur le pessimisme supposé des deux artistes, et s'oppose à la lecture optimiste que Kubrick entendait donner de la fin de la nouvelle :

Arthur Schnitzler voulait nous réveiller. Il n'est pas certain que Stanley Kubrick ait poursuivi ce but. [...] Arthur Schnitzler persistait à être un Aufklärer convaincu, un homme des Lumières ; Stanley Kubrick s'inscrit, lui, dans la lignée brillante des grands créateurs pessimistes 22 .

Au cœur de ce débat polémique sur les mérites respectifs de l'homme de lettres et du cinéaste, on voit se dessiner trois grandes lignes dans l'image de Schnitzler véhiculée par la réception d'Eyes Wide Shut.

\section{Schnitzler, auteur freudien}

La première reconduit le cliché simplificateur de Schnitzler en illustrateur des théories de Freud et de son Interprétation du rêve (Traumdeutung). Il est ainsi volontiers présenté comme « le double de Freud $»^{23}$. Jean-Pierre Dufreigne, dans son hommage au cinéaste, "Kubrick, les yeux grands fermés », évoque ainsi l'intrigue du film : «Dérapages de l'amour par temps de chien, d'après La Nouvelle rêvée d'Arthur Schnitzler, ce romancier autrichien, ce Freud de la fiction $»^{24}$. Le Journal du dimanche présente quant à lui ce portrait succinct : "Schnitzler, Autrichien, médecin avant d'être auteur dramatique, contemporain et ami de Sigmund Freud, fut l'écrivain de la cruauté mentale, travers qu'il se plut à disséquer dans nombre d'écrits $»^{25}$.

20. - Libération, $\mathrm{n}^{\circ} 5644,12$ juillet 1999, p. 33.

21. - Double rêve, traduit et présenté par Pierre Deshusses, Paris, Rivages poche, «Petite bibliothèque », 2010, p. 7.

22. - La Nouvelle rêvée, édition préfacée et annotée par Brigitte Vergne-Cain et Gérard Rudent, traduction nouvelle et présentation par Philippe Forget, Paris, Le Livre de poche, 1991, p. 7.

23. - Première, $\mathrm{n}^{\circ} 270$, septembre 1999, p. 69.

24. - L'Express, n²488, du 11 au 17 mars 1999, p. 117.

25. - Le Journal du dimanche, $\mathrm{n}^{\circ} 2741,11$ juillet 1999, p. 25. L'encart est signé C. G. 
Louvrage de Diane Morel, tributaire du récit de Raphael, livre une présentation de Schnitzler qui demeure elliptique :

Proche de la pensée de Freud, qui, sans l'avoir rencontré, le considérait comme son double en littérature, Schnitzler a déjà inspiré le cinéma, notamment avec La Ronde, court roman [sic] qui inspira le film éponyme à Max Ophuls pour lequel Kubrick a maintes fois dit son admiration. La nouvelle qui inspire Eyes Wide Shut [...] fait partie de la dernière période de l'artiste, la plus sombre, la plus pessimiste ${ }^{26}$.

L'essayiste minimise par ailleurs l'apport de Schnitzler au film, arguant que le projet kubrickien est « autant l'adaptation de la nouvelle de Schnitzler qu'une tentative de traiter le sujet de l'"inquiétante étrangeté" ${ }^{27}$.

Les critiques qui comparent véritablement les enjeux respectifs du livre et du film produisent des analyses plus nuancées : ainsi, Jean-Michel Frodon, dans "Stanley Kubrick, un démiurge parmi les hommes », salue « un grand film relativiste », tout en soulignant les subtilités du texte : " Alors que le texte [...] est hanté par le freudisme et par un mystère devant lequel les personnages finissent par s'incliner, reculant devant l'incompréhensible de la mort, du désir et de la psyché, le film choisit une version totalement désacralisée ${ }^{28}$; Pascal Mérigeau, dans l'article "Classique et baroque à la fois », prononce aussi l'éloge d'un « film humaniste » qui sonde à ses yeux l'héritage conjoint de Freud et Schnitzler :

Le court roman de Schnitzler à l'origine du film a été écrit au temps de la psychanalyse balbutiante. Kubrick [...] a pu y voir un matériau parfait pour étudier les effets d'un siècle, ou presque, de psychanalyse. Le siècle finissant fut celui de la psychanalyse et du cinéma. Comment l'une a-t-elle influé sur l'autre, comment l'autre s'est-il nourri de la science ? Ce sont deux des questions qui fondent le film ${ }^{29}$.

Dans un entretien intitulé « Kubrick rétablit la puissance du fantasme », la psychanalyste Élisabeth Roudinesco, estimant que la question de l'adultère n'est guère plus anodine à la fin du $\mathrm{Xx}^{\mathrm{e}}$ siècle que du temps de Schnitzler, ne limite pas davantage le texte schnitzlérien à une illustration servile de la Traumdeutung, mais en souligne la complémentarité et l'originalité :

26. - Diane Morel, Eyes Wide Shut ou l'étrange labyrinthe, Paris, PUF, « Rectoverso », 2002, p. 29-30.

27. - Ibid., p. 106.

28. - Le Monde, $\mathrm{n}^{\circ} 16944,19$ juillet 1999, p. 17.

29. - Le Nouvel Observateur, $n^{\circ} 1818$, du 9 au 15 septembre 1999, p. 22. 
On sait que Freud avait écrit une lettre à Schnitzler, lui expliquant qu'il ne voulait pas lire ses écrits car il craignait d'y retrouver son double... [...] Schnitzler était lui-même médecin, il admirait Freud sans pour autant adhérer à toutes ses thèses, et sa littérature se nourrit des grands thèmes de la psychanalyse, le rêve, le désir, la quête de soi, la mort...

La Nouvelle rêvée, qui traite de la connexion entre rêve et réalité, a été publiée [...] plus de vingt ans après L'Interprétation des rêves de Freud. La thématique est voisine, et l'influence incontestable, mais il est difficile d'être plus précis. Disons que Schnitzler et Freud sont les produits de la même culture, celle de la Vienne fin-de-siècle et de la médecine de l'époque. Cela donne d'un côté un écrivain et de l'autre un savant. On peut d'ailleurs imaginer que certains personnages de Schnitzler ont été des patients de Freud, l'un en tirant des études de cas, l'autre des romans. [...]

Ce qu'il y a de nouveau à la fois chez Freud et chez Schnitzler, c'est que [...] le rêve est l'accomplissement d'un désir inconscient. Mais de façon plus ou moins masquée, comme dans un rébus... 30

Dans les analyses qui ne se contentent pas de réduire la nouvelle à un palimpseste freudien, on voit ainsi émerger un deuxième topos, plus stimulant, qui dépeint Schnitzler en représentant emblématique de « l'apocalypse joyeuse ».

\section{Schnitzler, d'une fin-de-siècle à l'autre}

Cette topique a pour effet de produire, dans la réflexion sur le film, une assimilation entre la Vienne du Tournant du siècle et le New York de la fin du Xxe siècle. Jean-Claude Loiseau loue ainsi en Eyes Wide Shut un film «paradoxal, intemporel », fidèlement adapté d'un «grand auteur du début du siècle [qui] avait fait œuvre de visionnaire $»^{31}$. L'identification des deux époques est parfois nourrie par une réflexion sur le rôle évocateur de la musique chez Kubrick : Antoine Pecqueur estime, assez paradoxalement, que le choix d'une valse d'un compositeur soviétique, Chostakovitch, pour rythmer la scène du bal «nous ramène vers le Vieux Continent du début du $\mathrm{Xx}^{\mathrm{e}}$ siècle, vers cet empire austro-hongrois dans lequel Arthur Schnitzler a écrit la Traumnovelle $»^{32}$. L'article « Kubrick. Et la lumière fut » de Jean-Pierre Dufreigne suggère le diagnostic d'une nouvelle décadence, en décrivant des «personnages [enfermés] dans [la] prison [des] pulsions. Flirt entre Alice et un gentleman hongrois, caricature du charme fin-de-siècle Mitteleuropa [...]. Flirt entre Bill et

30. - Télérama, n²592, du 18 au 24 septembre 1999, p. 34-35.

31. - Ibid., p. 32.

32. - Antoine Pecqueur, Les Écrans sonores de Stanley Kubrick, Montdidier, Éditions du point d'exclamation, 2007, p. 11 et 14. 
deux top models, égéries obligées de notre fin-de-siècle $»^{33}$. Plus radicalement, l'essayiste Jordi Vidal voit en Eyes Wide Shut un " conte moral » apocalyptique sur une société capitaliste faite de simulacres et de rapports de domination; soulignant les effets de miroir qui ponctuent le film, qui évoquent peut-être le rapport de l'œuvre à sa source textuelle, il écrit :

[Durant la scène de bal], Alice [...] se fait courtiser par un vieux beau qui cabotine et sur-joue la fin de l'empire austro-hongrois sur le modèle dominant de l'inculture télévisuelle. Alice, absente par le regard et la parole, danse et tourne sur elle-même. À la manière de Max Ophuls, la caméra de Kubrick enserre le couple, comme s'il pouvait les confondre avec le monde d'avant la perte, et cette ronde qui les emporte mime le tourbillon viennois et pousse l'arrogance et la médiocrité jusqu’à évoquer le glissement du sens de Vienne à New York. [...] La boucle musicale qui l'emporte est comme la métaphore d'une Vienne sinistrée, d'où Musil, Schnitzler et Zweig auraient été bannis, et dont ne subsisterait de Freud que la névrose comme apologie du monde ${ }^{34}$.

La presse spécialisée poursuit ces variations sur le même thème, en établissant aussi fréquemment un parallèle, nourri de références cinématographiques, entre le New York de studio kubrickien et la Vienne décadente de Schnitzler. Nicolas Saada (« Scènes de l'envie conjugale ») insiste sur la «translation » opérée par le film :

Alice, un peu ivre, se laisse séduire par un gentleman hongrois à l'accent prononcé, qu'on croirait tout droit sorti d'un Lubitsch des années 30. Ophuls, Lubitsch : l'esprit de la Mitteleuropa s'immisce dans ce décor d'intérieur new-yorkais plus européen qu'américain. L'intelligence du travail d'adaptation de Kubrick a consisté à trouver des équivalences cinématographiques à l'écriture et au style dépouillé et fascinant de Schnitzler ${ }^{35}$.

Selon Michael Henry, dans «Eyes Wide Shut. La pénombre des âmes », Kubrick, soucieux de maintenir à l'écran l'ambiguiité fantastique de la nouvelle et d'interroger les dangers de l'imagination fantasmatique, se révèle être, à l'instar d'un Schnitzler qu'il compare sur ce point à Pascal, un «moraliste» :

La Vienne sulfureuse, fin-de-siècle, de Traumnovelle était bien faite pour le séduire [...]. Dans toute son œuvre, Arthur Schnitzler déploie les splendeurs et misères d'une capitale exsangue, langoureusement

33. - L'Express, n²514, du 9 au 15 septembre 1999, p. 83.

34. - Jordi Vidal,Traité du combat moderne. Films et fictions de Stanley Kubrick, Paris, Allia, 2005, p. 119-123.

35. - Les Cahiers du cinéma, n538, septembre 1999, p. 33. 
romantique, mais pourrie de l'intérieur, gangrenée par l'antisémitisme, travaillée par des forces maléfiques, à l'image d'un empire austro-hongrois qui est sur le point de se disloquer. Qui habite une telle poudrière paraît voué à une double vie, écartelé entre l'apparence et la réalité. Ce n'est pas un hasard si Freud vérifia certaines de ses intuitions dans les créations romanesques de son ami Schnitzler. Pas un hasard non plus si ce texte de 1926 est contemporain d'un cinéma germanique hanté par le dédoublement $[\ldots]$.

Kubrick lui-même se révèle en profonde sympathie avec la Stimmung austro-hongroise. Son Manhattan somnambulique se meut au ralenti, dans la durée savamment dilatée de certains films muets. [...] Voilà un cinéma envoûté, plus proche de Murnau et de Pabst que de nos réalisateurs contemporains [...]. Serait-ce que par un effet de palimpseste, [...] la Vienne des années vingt ne cesse de ressurgir comme le refoulé de New York ?36

L'analyse de Jean-Pierre Coursodon intitulée «Eyes Wide Shut. Fear and desire : la nuit des masques » souligne l'atemporalité d'un « récit d'apprentissage » ambigu, construit pour « se contester » lui-même, et liquide en ces termes le procès qu'il juge inutilement intenté à « l'anachronisme inévitable de la transposition »:

Un siècle sépare [...] l'épopée semi-onirique [du] couple schnitzlérien de sa version kubrickienne [...]. Cette distance ne peut manquer de soulever la question du bien-fondé de la modernisation [...].

On peut $[\ldots]$ aussi bien soutenir que tout ou presque, a changé, ou que tout ou presque reste pareil. Dans la mesure où la préoccupation essentielle de Kubrick [...] est la vie érotique de ses personnages, son refus de reconnaître les changements survenus par ailleurs dans les rapports entre sexes paraît paradoxalement justifié, l'imaginaire érotique étant ancré dans des archétypes finalement peu sensibles à l'évolution des mœurs et des modes. [...] Il semble [d'ailleurs que Schnitzler lui aussi] ait entretenu à plaisir l'incertitude sur l'époque [...], de même qu'il entretient l'incertitude sur la nature (rêves, fantasmes, réalité ?) des faits rapportés ${ }^{37}$.

Michel Ciment, grand spécialiste de Kubrick, poursuit cette légitimation de l'adaptation de Schnitzler à l'écran par une réflexion sur les paradoxes des fins-de-siècle, marquées tout à la fois par une effervescence de la pensée et la conscience d'un déclin imminent :

la Vienne fin-de-siècle, celle de la Joyeuse Apocalypse, où naissent la sociologie moderne et la psychanalyse, où l'effondrement de l'empire des Habsbourg secoue autant les fondements de l'Europe qu' un siècle aupa-

36. - Positif, n463, septembre 1999, p. 6-11.

37. - Ibid., p. 12-15. 
ravant la Révolution française, offre comme une reprise, sur un mode plus sombre, du climat de l'Aufklärung finissant. Kubrick ne pouvait qu'être sensible à cette vision du monde où les ambiguittés de la nature humaine (bien et mal inextricablement mêlés) répondent à l'inquiétante ambivalence de l'univers. [...] [Chez Zweig et Schnitzler], Kubrick retrouve des libres penseurs comme lui, des sceptiques mais aussi des esprits critiques face aux valeurs établies. Dans la Vienne du Jugendstil et de la Sécession s'incarne la modernité. [...] Le goût de la tradition et la volonté de rompre avec elle, qui sont au cœur de la culture viennoise, sont aussi des traits essentiels de la personnalité du metteur en scène. Carl Schorske [...] a pu voir en Schnitzler un artiste chez qui se retrouvent à égales proportions préoccupations morales et scientifiques et recherches esthétiques, ce qui n'est pas si éloigné de la démarche kubrickienne. La conscience qu'avait l'écrivain autrichien de la nécessité de prendre en compte les instincts vitaux, comme son sentiment des souffrances qu'ils pouvaient infliger aux autres, courent aussi en filigrane dans l'œuvre de Kubrick [...].

Transposer Rien qu'un rêve de la Vienne cosmopolite de 1900, où la communauté juive jouait un rôle important, dans le New York non moins pluriethnique d'aujourd'hui, substituer à la capitale de l'empire des Habsbourg la ville phare d'un empire américain tout-puissant n'a donc rien d'artificiel ${ }^{38}$.

Incarnation d'une époque, Schnitzler semble pourtant peiner à affirmer dans ces pages une identité irréductible à toute comparaison.

\section{Schnitzler, un auteur sans visage propre?}

Le troisième stéréotype qui émane de la critique consacrée à Eyes Wide Shut identifie la figure du cinéaste à l'auteur autrichien. La recension négative de Jean Roy (" Les yeux grands ouverts de Kubrick ») introduit ironiquement cette hypothèse : « libre à chacun de repérer que Tom Cruise incarne un médecin, comme Schnitzler, son père ou le père de Kubrick ${ }^{39}$. De même, attribuant encore à Schnitzler un masque freudien, François Gorin, dans un article très réservé envers le film, qu'il juge trop inféodé au livre (« La dernière tentation de Kubrick »), donne cet aperçu sélectif de l'œuvre et de la personnalité de Schnitzler :

Ce récit [...] appartient à la veine fantastique du dramaturge viennois, qui consignait ses rêves par dizaines dans son Journal et que Freud évita longtemps par crainte « de rencontrer [son double] ». [...] [Cette histoire] obsède depuis longtemps un Stanley Kubrick qui, fils de

38. - Michel Ciment, Kubrick, op. cit., p. 263-264.

39. - L'Humanité, nº17133, 15 septembre 1999, p. 21. 
médecin comme Schnitzler, faillit devenir lui-même médecin comme le fut l'écrivain ${ }^{40}$.

Michel Chion, autre spécialiste de Kubrick, reprenant nombre des caractéristiques souvent conférées à Schnitzler, rappelle quant à lui le contexte de « l'apogée culturelle » viennoise dans lequel évoluait l'auteur autrichien qui, « comme Kubrick, était un juif non pratiquant et fils de médecin », « souvent qualifié de freudien » « à cause de la présence du rêve dans son œuvre »; il souligne la pertinence de la comparaison implicite établie par le réalisateur entre le New York contemporain et la Vienne tout aussi « permissive » de Schnitzler, avant de remarquer que Kubrick commença de travailler à ce film à 63 ans, l'âge où Schnitzler écrivit la Traumnovelle, « un texte d'une extraordinaire richesse, un défi pour un cinéaste ${ }^{41}$.

L'analyse foisonnante que livre Jean-Paul Enthoven dans son article «Les yeux grands ouverts du docteur Schnitzler » semble finalement réunir tous les traits attribués à l'auteur viennois par la critique kubrickienne. Dans cet éloge d'un film désigné comme une « fable », il ne regrette que le renoncement du cinéaste à traiter l'enjeu de l'antisémitisme dont «Schnitzler, médecin juif, avait une longue expérience », et donne de lui une présentation particulièrement vivante, nourrie par une série de comparaisons avec ses frères en écriture :

Derrière les images glacées du film de Stanley Kubrick, il y a [...] les mots brûlants d'un texte fort bref [...]. Derrière le New York de Park Avenue et du Village, il faut donc guetter un spectre viennois. Et derrière les soirées échangistes qui troublent l'aimable Tom Cruise, on entendra, tel un écho de " joyeuse Apocalypse », ce crépuscule austro-hongrois dont le génial Arthur Schnitzler [...] s'était fait, au début du siècle, l'explorateur mélancolique. D'ailleurs, cet homme si singulier - il fut romancier, médecin, dramaturge, noceur - adorait le cinéma. Il y devinait un art neuf et propice à l'ellipse, à l'allusion, au vague, qui furent ses tonalités de base. Kubrick, paraît-il, parlait de cet écrivain, si proche de Tchekhov et de Tourgueniev, comme d'un frère trop vite disparu. Un frère bizarre et sensuel. Un expert en pessimisme et en duperie amoureuse.

Il est vrai que le docteur Schnitzler, en son temps, sut séduire la plupart des êtres qui traversèrent son existence. Les femmes, tout d'abord, qu'il considéra comme un gisement inépuisable de fiction. [...] Auprès d'elles, il prit des leçons de manque et d'insatisfaction - dans Traumnovelle, son hérö̈ne ne porte-t-elle pas, en souvenir de Proust, le prénom d'Albertine ? Mais Schnitzler assura son autre réputation du côté de l'esprit. À Vienne, on se le disputait dans les dîners, dans les cafés,

40. - Télérama, n²584, du 24 au 30 juillet 1999, p. 29.

41. - Michel Chion, Stanley Kubrick. L'humain, ni plus ni moins, Paris, Cahiers du cinéma, 2005, p. 452-461 et 510 . 
dans les revues. On adorait son intelligence paradoxale et sensible. On fit un triomphe à son théâtre - alors qu'il ne s'illustra dans ce genre, de son propre aveu, qu'afin de rencontrer de jeunes actrices. Cet homme - auquel Freud écrivit : "J'ai toujours eu l'impression que vous étiez mon double » - bâtit ainsi son œuvre autour de la sexualité, du corps, de l'interdit. Il revendiquait, sur ces affaires, des yeux grands ouverts ${ }^{42}$.

Dans ce portrait en mosaïque, le visage de Schnitzler, toujours insaisissable, semble disparaître parmi ses multiples alter egos...

\section{Schnitzler et ses masques}

La réception schnitzlérienne portée par celle d'Eyes Wide Shut, rarement fondée sur des références précises au texte de la Traumnovelle, constitue une illustration supplémentaire du rôle crucial des intermédiaires d'une œuvre, qui en facilitent la diffusion ou au contraire en biaisent ou freinent la compréhension. L'image de Schnitzler qui se dégage des analyses du film semble ainsi souvent filtrée par la reconduction de lectures partiales ou même fautives, même si l'assimilation de cet auteur, qui semble avancer masqué, à une époque entière et à toute une galerie de penseurs et d'artistes produit incontestablement des lectures stimulantes de son œuvre.

42. - Le Point, $\mathrm{n}^{\circ} 1408,10$ septembre 1999, p. 103. 
